\title{
Dynamic scaling of $I-V$ data for the neutral 2D Coulomb gas
}

\author{
Stephen W. Pierson ${ }^{\mathrm{a}, 1}$, Mark Friesen ${ }^{\mathrm{b}}$ \\ a Department of Physics, Worcester Polytechnic Institute, Worcester, MA 01609-2280, USA \\ b Applied Superconductivity Center, University of Wisconsin, Madison, Wisconsin 53706, USA
}

\begin{abstract}
The value of the dynamic critical exponent $z$ has been studied for experimental two-dimensional superconducting and Josephson Junction array systems in zero magnetic field via the Fisher-Fisher-Huse dynamic scaling analysis. We found $z \simeq 5.6 \pm 0.3$, a relatively large value indicative of non-diffusive dynamics. We extend this work here to simulational $I-V$ curves that are also found to be characterized by the same large value of $z$.
\end{abstract}

Keywords: Kosterlitz-Thouless dynamics; Dynamic Scaling; I-V characteristics

Dynamical scaling is a powerful tool for analyzing the dynamic critical behavior[1] of a variety of systems. Fisher, Fisher and Huse, (FFH)[2] have derived a dynamic scaling form for superconducting systems that should apply under a variety of conditions, from zero applied magnetic field to finite field, from "clean" to disordered systems, as long as the transition is continuous. Typically, the "FFH" scaling is applied to finite field $I$ - $V$ curves.

Recently the authors of this manuscript applied the FFH dynamic scaling to zero-field experimental data from two-dimensional (2D) superconducting films, Josephson junction arrays (JJA's), and superfuid ${ }^{4} \mathrm{He}$ films[3,4] to find a value $z \simeq 5.6$ for the dynamical critical exponent. The commonly expected and reported value of $z$ for these systems is 2 , which represents simple diffusion. In this paper, we extend our work to simulational $I$ - $V$ data

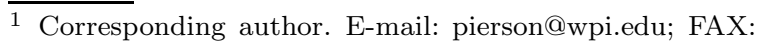
(508) 831-5391 on the neutral 2D Coulomb gas and find $z \simeq 5.6$.

The traditional form of the FFH scaling is $V=$ $I \xi^{-z} \chi_{ \pm}(I \xi / T)$, where $\chi_{+(-)}(x)$ is the scaling function for temperatures above (below) the transition temperature $T_{c}$ and $\xi$ is the vortex correlation length. We use a form that is slightly different:[5]

$$
I^{1+1 / z} /\left[T V^{1 / z}\right]=\varepsilon_{ \pm}(I \xi / T)
$$

where $\varepsilon_{ \pm}(x) \equiv x / \chi_{ \pm}^{1 / z}(x)$. The left-hand side of this equation does not involve any factors of $\xi$, which tend to stretch the scaling axes, thereby diminishing the resolution. Thus Eq. (1) allows one to better judge the scaling, as we mention below.

We demonstrate the application of Eq. (1) for the numerical data of Lee and Teitel[6]. Using a finite-size scaling analysis, those authors found a dynamical exponent of $z=2$. The parameter $C=$ 0.35 entered through the FFH scaling using the conventional form for the Kosterlitz-Thouless correlation length, $\xi=\exp \left[C /\left|T-T_{c}\right|^{1 / 2}\right]$, while the transition temperature, $T_{c}=0.218$, was deter- 
mined in equilibrium simulations[6]. The results are shown in Fig. 1. The collapse is not convincing, especially for the $T<T_{c}$ isotherms. (It is instructive to contrast Fig. 1 with the Fig. 5 of Ref. [6], where the scaling is presented for the same parameter values but in the original FFH dynamic scaling form. This comparison illustrates the advantage of using Eq. (1) to judge the scaling collapse.)

By varying the scaling parameters $T_{c}, z$, and $C$ in a least-squares minimization of the separation between scaled data, we have obtained a more convincing collapse of the Lee-Teitel data, as shown in Fig. 2, curve (a). According to the dynamical scaling hypothesis of $\mathrm{FFH}$, the critical isotherm, $T=T_{c}$, is expected to form a perfect power-law, $V \propto I^{z+1}$, thus appearing straight in a log-log plot of $I$ and $V$. Generally, it is preferable to identify $T_{c}$ in this way, permitting only small variations from this estimate in an $I-V$ fitting procedure[3,4]. However, according to the power-law criterion, the Lee-Teitel data all appear to lie in the high temperature range, $T>T_{c}$. We therefore allow $T_{c}$ to remain an unrestricted parameter, obtaining the fitting results $T_{c}=0.122, z=5.6$, and $C=1.13$. We performed a similar analysis on the Monte Carlo data of Weber et al.[7] and found an optimal collapse for $T_{c}=0.131, z=5.6$, and $C=1.463$, as shown in Fig. 2, curve (b). These values of $T_{c}$ and $z$ are combatible with the power-law criterion.

The similarities between scaling results in experimental systems $[3,4]$ and the simulations studied

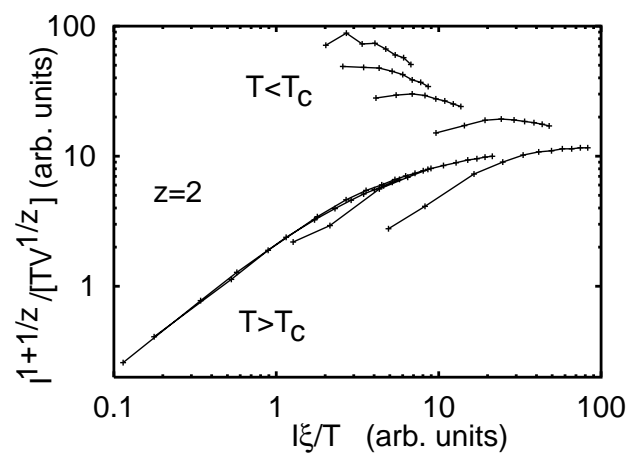

Fig. 1. Simulational $I-V$ data of Lee and Teitel[6], scaled according to Eq. (1), with $z=2$. The scaling parameters were determined in Ref. [6], using finite-size scaling.

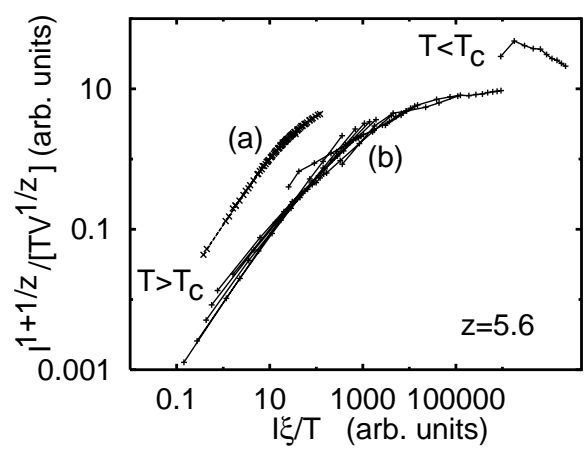

Fig. 2. (a) The Lee-Teitel $z=5.6$ results[6]; and (b) the Weber et al. $z=5.6$ scaling results[7].

here are striking. In every case, optimization produces unexpectedly high, but universal values of $z \simeq 5$.6. However, the results presented here raise some important questions. It is apparent that the critical $I$ - $V$ isotherm, separating superconducting from nonsuperconducting behavior, occurs significantly below the equilibrium $(I=0)$ transition temperature. It is not yet clear whether this lower transition temperature is an artifact of finite-size effects, or represents a more fundamental phenomenon in $2 \mathrm{D}$ vortex dynamics. We intend to address this issue in the future.

\section{Acknowledgement}

SWP acknowledges the The Petroleum Research Fund, administered by the ACS, for their support.

\section{References}

[1] P. C. Hohenberg and B. I. Halperin, Rev. Mod. Phys. 49 (1977) 435.

[2] D. S. Fisher et al., Phys. Rev. B 43 (1991) 130.

[3] S. M. Ammirata et al., Physica C 313 (1999) 225.

[4] S.W.Pierson et al., Phys.Rev.B 60, 1309 (1999).

[5] Other scaling ansatz [e.g. R. A. Hyman et al., Phys. Rev. B 51, 15304 (1995)] lead to slight quantitative differences with the results given here, but do not affect our conclusions.

[6] J.-R. Lee and S. Teitel, Phys. Rev. B 50 (1994) 3149.

[7] H. Weber et al., Phys. Rev. B 53 (1996) 8566. 